\title{
Contribution to the Theory of Periodic Reaction of Three Bodies Systems
}

\author{
Yasuhiro Suzuki \\ Graduate School of Informatics, Nagoya University, Furocho Chikusa \\ Nagoya City, Aichi Prefecture 464/0814, Japan*
}

E-mail: ysuzuki@nagoya-u.jp.com

www.ysuzuki.info

\begin{abstract}
The chemical ecosystem is an ecosystem in which chemical substances mediate interactions. When herbivores feed, the plant analyzes herbivore saliva and produces volatile chemicals that attract herbivore natural enemies. Natural enemies are attracted to volatile chemicals and eliminate herbivores. This system is a tripartite system consisting of herbivore, carnivore and volatile chemicals. The basic equation of mathematical ecology is the Lotoka-Volterra equation. This equation is a two-way system of herbivores and carnivores. This paper proposes the basic equations of three chemical ecosystems and shows their mathematical features.
\end{abstract}

Keywords: Chemical Ecology, Lotoka-Volterra equation, Tritrophic system, mathematicalbiology

\section{Introduction}

Mathematical ecology has used the Lotka-Volterra equation as the basic equation ${ }^{1)}$. This equation is a simultaneous differential equation with the predator as $\mathrm{y}$ and the prey as $\mathrm{x}$.

$$
\begin{aligned}
& \frac{d x}{d t}=k_{1} x-k_{2} x y, \\
& \frac{d x}{d t}=k_{1} x-k_{2} x y,
\end{aligned}
$$

The equilibrium points of this simultaneous differential equation are obtained from ${ }^{3)}$ and ${ }^{4)}$

$$
\begin{aligned}
& 0=\frac{d x}{d t}=x\left(k_{1}-k_{2} y\right), \\
& 0=\frac{d y}{d t}=y\left(k_{2} x-k_{3}\right),
\end{aligned}
$$

as

$$
(x, y)=\left\{(0,0),\left(\frac{k_{3}}{k_{2}}, \frac{k_{1}}{k_{2}}\right)\right\}
$$


Therefore, if $y$ is larger than $\mathrm{k}_{1} / \mathrm{k}_{2}$, $\mathrm{x}$ decreases, and if $\mathrm{y}$ is smaller, it increases. On the other hand, if $\mathrm{x}$ is larger than $\mathrm{k}_{3} / \mathrm{k}_{2}$, $\mathrm{y}$ increases, and if it is smaller, $\mathrm{y}$ decreases. Therefore, $\mathrm{x}$ and $\mathrm{y}$ oscillate around the equilibrium point. The solution to this equation is periodic ${ }^{1)}$ (Fig. 1). The Lotoka Volterra equation is a basic equation of mathematical ecology, and various variants have been proposed (such as ${ }^{2)}$ ).

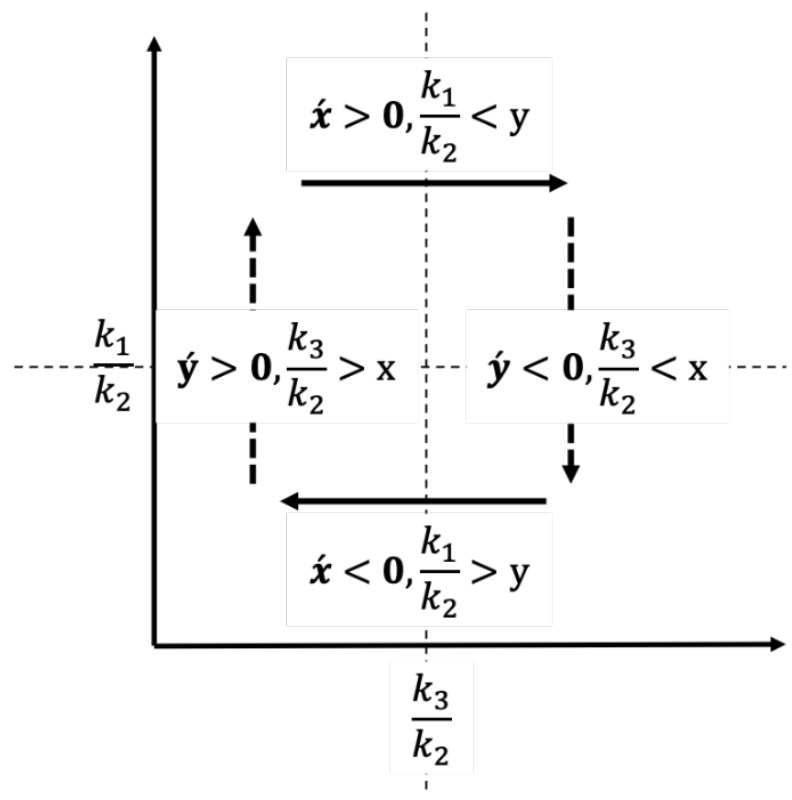

Fig. 1. The isocline analysis of LV equation

\subsection{Chemical Ecology}

Plants are the foundation and resources that support the entire ecosystem, but on the other hand, indirect defense using pests and communication between plants have been revealed. [3]

Since the late 1980s, it has become clear that plants attract predators' natural enemies by releasing volatile substances in response to feeding damage [3]. In this case, there is an indirect interrelationship through odor information between plants and natural enemies that are not in an "eat-eat" relationship that goes beyond the nutritional stage. Plant-predators-predators span three nutritional stages and are linked not only in the food chain but also in exchanging information.

Volatility produced by plants is called Herbivore Induced Plant Volatiles (HIPV). When pests are directly exterminated using pesticides, the side effect is to exterminate the pests to be exterminated and the natural enemies existing in the space. In that case, if pests invade the place where the natural enemy was exterminated again, the natural enemy cannot control it. As a result, plants suffer great feeding damage. To avoid this, it is necessary to spray pesticides over a wider area, and the ecosystem will be seriously damaged.

On the other hand, if a plant that has been damaged by feeding produces HIPV and provides indirect defense by natural enemies, the ecosystem will not be seriously damaged. Plants have their natural enemies exterminate pests in return for the cost of producing scents. Natural enemies can increase the efficiency of finding pests in return for the cost of learning scents. However, pests are natural enemies if they eat plants. It is unilaterally disadvantageous because it is eaten by.

In reality, pests are not unilaterally disadvantaged. Plants do not produce HIPV immediately after being damaged by feeding. Plants do not produce HIPV until they are about to die. Therefore, there is time to spare from the start of feeding to the invasion of natural enemies. Adult females spawn until the invasion of natural enemies and move to other safe strains before invading natural enemies. Therefore, pests get a space where natural enemies do not exist in return for the cost of attracting natural enemies and it can lay eggs safely.

\subsection{Chemical Ecology, rule based expression}

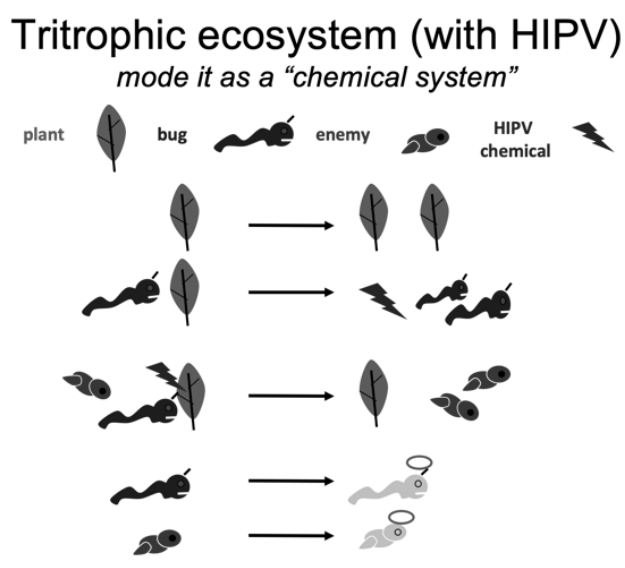

Fig. 2. Schematic rule based model of the tritrophic system composed of Plant-Herbivores and Carnivore 
Describe the chemical ecosystem on a rule based (Fig.2). The rule base is an abstract chemical reaction system. An abstract chemical reaction system is a method of describing a phenomenon using the notation of a chemical formula. Reaction kinetics transforms chemical equations into differential equations.

Chemical species used in chemical ecosystems are plants, prey, predators and HIPV.

$$
\text { Plant + Predator-> Prey, Predator, HIPV }
$$

This reaction expresses that the prey (herbivore) eats the plant to increase the population and the plant produces HIPV.

$$
\text { HIPV + prey (carnivorous) + predator-> predator, }
$$
predator

This reaction expresses that HIPV-induced predators eat prey and increase populations. Plants cease to produce HIPV as there are no more herbivores. Therefore, there is no HIPV on the right side of this equation.

\section{Predator->}

This reaction represents the natural death of a predator. The reason for not including the natural death of prey is that the number of prey is decreasing too much. Counting the increase and decrease of predators with the above three chemical formulas, it is 0 . The increase / decrease in predators is 0 . Therefore, the increase / decrease in prey and predator is balanced. If the natural death of the prey is added, the prey will decrease and become extinct even if it is not predated. Its extinction is not essential, but the balance of stoichiometry. On the other hand, without the natural death of predators, the number of predators increases exponentially. This increase is also a problem of stoichiometric balance and is not essential. Therefore, there was no natural death of the predator, and only the predator died of natural causes.

\section{Basic equation of chemical ecosystem}

Chemical ecosystems are not just related to prey and predators. An information chemical called HIPV intervenes between prey and predators. The indirect interaction between prey and predator by HIPV is the essence of the chemical ecosystem. Therefore, the chemical ecosystem is an interaction of three bodies.

The Lotka-Volterra equation cannot describe the chemical ecosystem in essence. Lotka-Volterra equations with multi-bodies have been proposed [4]. The $\mathrm{n}$ species Lotka-Volterra equation has two or more predators or prey but they are essentially two-body interaction, not an $\mathrm{n}(\mathrm{n}>2)$ bodies interactions. Therefore, the generalized Lotka-Volterra equation cannot essentially describe the chemical ecosystem.

Let $\mathrm{x}$ be the number of prey, $\mathrm{y}$ be the predator, and $\mathrm{i}$ be the information (HIPV). $\mathrm{x}, \mathrm{y}, \mathrm{i}$ are all positive numbers greater than or equal to 0 .

Prey increases by eating plants and decreases by predation by predators. Unlike the LV equation, when a prey eats a plant, the plant produces HIPV, which is represented by i. Predators cannot find any prey if the density of HIPV is low. In addition, the number of prey is reduced by being preyed on by predators. HIPV plays the role of stabilizer, predators cannot have prey as much as possible. Because, if predators get many preys, the concentration of HIPV becomes low and predators cannot find preys. The basic equation of the chemical ecosystem based on the LV equation can be obtained from the above.

$$
\begin{aligned}
& \frac{d x}{d t}=k_{1} x-k_{2} x y i, \\
& \frac{d y}{d t}=k_{2} x y i-k_{3} y, \\
& \frac{d i}{d t}=k_{1} x-k_{2} x y i .
\end{aligned}
$$

What makes this equation essentially different from the LV equation is that it includes a term for three-body interactions.

\section{Behavior of basic equations of chemical ecosystems}

The equilibrium points of this equation are

$$
0=\frac{d x}{d t}=x\left(k_{1}-k_{2} y i\right),
$$




$$
\begin{aligned}
& 0=\frac{d y}{d t}=y\left(k_{2} x i-k_{3}\right), \\
& 0=\frac{d i}{d t}=x\left(k_{1}-y k_{2}\right),
\end{aligned}
$$

$$
\text { and }(x, y, i)=\left\{(0,0,-),\left(\frac{k_{3}}{i k_{2}}, \frac{k_{1}}{i k_{2}}, \frac{k_{1}}{k_{2}}\right)\right. \text {. }
$$

The $L V$ equation has two equilibrium points, but in this equation the equilibrium points are all points on $\mathrm{c} / \mathrm{i}$ and are infinite. And when $\mathrm{i}=1$, the equilibrium point coincides with the LV equation. When $\mathrm{i}=0$, $\mathrm{x}$ and $\mathrm{y}$ have no equilibrium point. When $\mathrm{i}=0, \mathrm{dx} / \mathrm{dt}>0$ and $\mathrm{dy} / \mathrm{dt}$ $<0$. If $\mathrm{k} 1, \mathrm{k} 2$, and $\mathrm{k} 3$ are positive constants, $\mathrm{k} 3 / \mathrm{k} 2$ is $\mathrm{c} 3$, and $\mathrm{k} 1 / \mathrm{k} 2$ is $\mathrm{c} 1$, the equilibrium point is $(\mathrm{c} 3 / \mathrm{i}, \mathrm{c} 2 / \mathrm{i}$, c2).

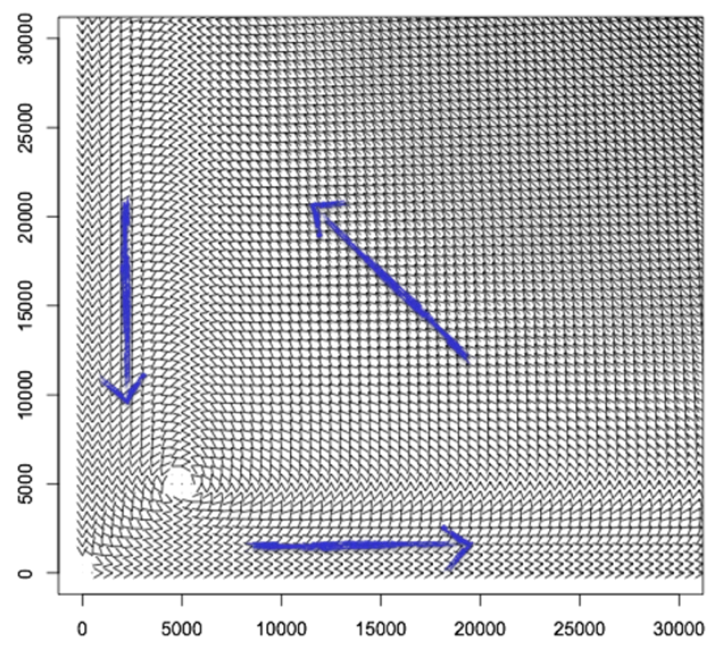

Fig. 3. Vector space of LV equation

\subsection{Vector space of equations}

We compared the behaviour of the trytrophic equations with the LV equation. So we investigated both vector fields. The LV equation's vector field is a periodic solution centred on the origin and another equilibrium point, and this result has been known for a long time (Fig. $3)$.

The diagonal is the set of equilibrium points. In reality, the equilibrium points on this diagonal are $\mathrm{k} / \mathrm{i}$ of the equilibrium point curve. Below this diagonal, the concentration of HIPV is low, so $\mathrm{Y}$ decreases and $\mathrm{X}$ increases. Above this diagonal, the concentration of HIPV is high, so Y increases and X decreases. Since the

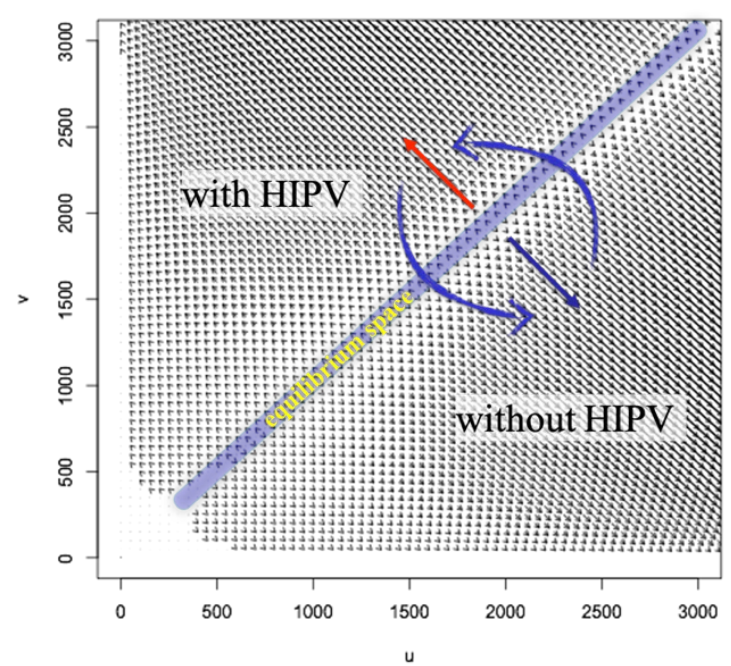

Fig. 4. Vector space of basic equation of tritrophic system., The diagonal is the set of equilibrium points. In reality, the equilibrium points on this diagonal are $\mathrm{k} / \mathrm{i}$ of the equilibrium point curve.

sum of these two vectors is exactly the opposite, the vector field shows that the tritrophic basic equation has a stable manifold structure. (Fig.4).

From this result, it is foreseen that the basic equation of tritrophic is significantly different from the LV equation and has an equilibrium solution. In the future, I would like to proceed with a more rigorous mathematical analysis.

\section{References}

1. Alfred J.Lotoka, Elements of Physical Biology, Williams and Wilkins Company, 1925.

2. Dimas Martins Afonso, Gjini Erida, Modeling Competitive Mixtures With the Lotka-Volterra Framework for More Complex Fitness Assessment Between Strains, Frontiers in Microbiology, 11, 2132, 2020.

3. Takabayashi J., Dicke M. (1997) Herbivore-Induced Plant Volatiles with Multifunctional Effects in Ecosystems: A Complex Pattern of Biotic Interactions. In: Abe T., Levin S.A., Higashi M. (eds) Biodiversity. Springer, New York, NY. https://doi.org/10.1007/978-1-4612-1906-4_9

4. Kerner, Edward H, Comment on Hamiltonian structures for the n-dimensional Lotka-Volterra equations, Journal of Mathematical Physics 38, 1218 (1997); https://doi.org/10.1063/1.5318751, Published Online: 04 June 1998 